\title{
COMMENT
}

\section{Update the WHO EML to improve global paediatric rheumatology}

Helen E. Foster ${ }^{1 *}$ and Christiaan Scott ${ }^{2}$

The World Health Organization (WHO) Essential Medicines List (EML) informs policy makers about which medications should be prioritized and is particularly important for countries with limited resources. However, the EML lacks vital medicines used in paediatric rheumatology, the inclusion of which could transform the lives of many children around the world.

An estimated 6-7 million children worldwide have rheumatic diseases ${ }^{1}$, many of whom live in the poorest parts of the world in Africa or Asia. Delays in access to the appropriate care for children with rheumatic diseases have been reported globally ${ }^{1,2}$, and a 2019 study reported worse outcomes for children with juvenile idiopathic arthritis (JIA) in countries with a low $\mathrm{GDP}^{3}$. The explanation is multi-factorial and includes delays in presentation and diagnosis and poor access to medicines (both availability and affordability), as well as workforce challenges ${ }^{4}$. To improve outcomes in low-resource and middle-resource income countries (LRICs and MRICs), the solutions are complex and include improved education of community health-care workers and the training of more paediatric rheumatologists ${ }^{2,4}$. Furthermore, there is need for improved access to the necessary medicines at a policy level by ensuring resources such as the World Health Organization (WHO) Essential Medicines List (EML) and EML for Children (EMLc) are up to date.

The WHO EML informs countries around the world about the minimum medicine items necessary to meet the most important priority health needs of a population (both adults and children) ${ }^{5}$. The first separate WHO EMLc was published in 2007 and places an emphasis on the importance of appropriate drug formulations for children. Both lists are aligned to the United Nations aim for Universal Healthcare Coverage and are integral to the WHO's approach to addressing the Sustainable Development Goals for 2030. The EMLs are also used to inform the national medicine lists of many countries, especially LRICs and MRICs.

The $2019 \mathrm{EML}^{5}$ includes a section on 'medicines for diseases of joints', which is divided into sub-sections for adults and children. Notably, under 'juvenile joint diseases', acetylsalicylic acid (aspirin) is the only medicine listed and is indicated for use in rheumatic fever, JIA or Kawasaki disease. By contrast, the 'adult joint diseases' section of the EML includes methotrexate, sulfasalazine and hydroxychloroquine for inflammatory arthritis. Several other relevant medications are listed elsewhere in the EMLs, including glucocorticoids, NSAIDs and some DMARDs, but no reference is made to their particular use in paediatric rheumatology.

The medications included in the current lists, along with their formulations and indications for use, do not fully address the needs of children with rheumatic diseases in the context of current approaches to management. An updated EMLc with improved clarity around the medicines for use in childhood-onset rheumatic diseases is therefore needed. For example, the inclusion of intra-articular glucocorticoids and appropriate formulations of methotrexate would facilitate access to an effective treatment for the majority of children with JIA. As Chair (H.E.F.) and Co-Chair (C.S.) of the Paediatric Global Musculoskeletal Task Force, we are working with the global paediatric rheumatology community to amend the sections of the forthcoming 2021 EMLc that are relevant to paediatric rheumatology; the process to submit a case of need for the proposed changes starts in early 2020 . We therefore have a golden opportunity to work together with the WHO to enable improved access to appropriate care for children with rheumatic diseases around the world.

1. Henrickson, M. Policy challenges for the pediatric rheumatology workforce: Part III. the international situation. Pediatr. Rheumatol. 9, 26 (2011).

2. Foster, H. E. \& Rapley, T. Access to pediatric rheumatology care - a major challenge to improving outcome in juvenile idiopathic arthritis. J. Rheumatol. 37, 2199-2202 (2010).

3. Consolaro, A. et al. Phenotypic variability and disparities in treatment and outcomes of childhood arthritis throughout the world: an observational cohort study. Lancet Child Adolesc. Health 3, 255-263 (2019).

4. Scott, C. et al. Juvenile arthritis management in less resourced countries (JAMLess): consensus recommendations from the Cradle of Humankind. Clin. Rheumatol. 38, 563-575 (2019).

5. World Health Organization. WHO Model Lists of Essential Medicines. WHO.int https://www.who.int/medicines/publications/ essentialmedicines/en/ (2019).

\section{Competing interests}

The authors declare no competing interests. 